\title{
Racial/Ethnic Minorities Ineligible for Direct Access Colonoscopy (DAC): Identifying Patients Who Fall Through the Cracks
}

\author{
Sarah J. Miller • Jamilia R. Sly • Steven H. Itzkowitz • \\ Lina Jandorf
}

Received: 10 April 2014 /Revised: 18 August 2014 / Accepted: 22 August 2014 /Published online: 2 October 2014

(C) W. Montague Cobb-NMA Health Institute 2014

\begin{abstract}
Objective Patients ineligible for direct access colonoscopy (DAC) are typically referred for a pre-colonoscopy consultation with gastroenterology (GI). However, the referral from primary care to GI creates the potential for patients to drop out of treatment. The primary objective of the current study was to examine the proportion of participants deemed ineligible for DAC that (1) attended an appointment with GI and (2) completed a screening colonoscopy. The second aim of the study was to examine predictors of screening colonoscopy adherence.

Methods Participants $(N=144)$ were average-risk patients who received a primary care referral for a screening colonoscopy and were deemed ineligible for DAC between 2008 and 2012. Following the primary care visit, participants completed a questionnaire that assessed demographics and psychological factors. Medical chart review determined whether participants completed the screening colonoscopy via the GI referral.

Results Of the 144 participants, only 19 (13\%) completed the screening colonoscopy via the GI referral. Multiple regression analyses revealed that decisional balance was the only unique predictor of screening colonoscopy adherence.

Conclusions Patients deemed ineligible for DAC are highly unlikely to complete a screening colonoscopy. Interventions
\end{abstract}

\section{S. J. Miller $(\bowtie) \cdot$ J. R. Sly $\cdot$ S. H. Itzkowitz $\cdot$ L. Jandorf}

Department of Oncological Sciences, Icahn School of Medicine at

Mount Sinai, One Gustave L. Levy Place, Box 1130, New York,

NY 10029-6574, USA

e-mail: Sarah.Miller@mssm.edu

J. R. Sly

e-mail: Jamilia.Sly@mssm.edu

S. H. Itzkowitz

e-mail: steven.itzkowitz@mountsinai.org

L. Jandorf

e-mail: Lina.Jandorf@mssm.edu are needed to increase screening colonoscopy adherence in this vulnerable population.

Keywords Colonoscopy · Minority health · Disparities · Direct access colonoscopy

\section{Background}

Although colorectal cancer (CRC) is a largely preventable disease, CRC remains the third leading cause of cancer death in the USA [1]. Regular CRC screening (e.g., colonoscopy) can aid in the early detection and prevention of CRC. Due to the proven effectiveness of CRC screening, published guidelines recommend that average-risk people begin regular CRC screening at the age of $50[2,3]$. Of the recommended screening tests, a colonoscopy is the preferred screening method because it can effectively detect and treat CRC [4]. It has been estimated that screening colonoscopies can prevent up to $65 \%$ of CRC incidence [1].

In an attempt to increase screening colonoscopy rates, many medical centers have developed programs to directly refer patients from their primary care provider (PCP) to the endoscopy suite in a process referred to as direct access colonoscopy (DAC). By avoiding a pre-procedure visit to the gastroenterologist, DAC reduces wait time before a colonoscopy and decreases the need for multiple clinic visits [5]. Over the last 15 years, the DAC referral system has become increasingly incorporated into standard clinical practice in the USA [6]. The New York Citywide Colon Cancer Control Coalition (C5) and the New York City Department of Health and Mental Hygiene (NYCDOHMH) developed a Direct Referral Initiative for screening colonoscopies [7]. Likewise, we have been using a DAC system at our institution since 2003 [8]. 
While DAC is widely viewed as enhancing the efficiency and timeliness of completing a colonoscopy, DAC is not appropriate for all patients. Patients may not be suitable for direct referral if, for example, they are currently taking an anticoagulation medication, have multiple co-morbidities or a history of problems with sedation/anesthesia, or have uncontrolled hypertension or diabetes. In order to help PCPs determine which patients may be inappropriate for DAC, the New York C5 and the NYCDHMH created a "Direct Referral for Screening Colonoscopy" checklist [7]. (For the complete Direct Referral for Screening Colonoscopy checklist, see http:// www.nyc.gov/html/doh/downloads/pdf/cancer/cpnp-dersform.pdf).

Patients who are deemed inappropriate for DAC are typically referred for a pre-colonoscopy consultation with a gastroenterologist to optimize their colonoscopy experience and attempt to mitigate adverse events. This additional referral requires patients to (1) schedule an appointment with GI; (2) attend the scheduled GI appointment; (3) schedule a screening colonoscopy; and then finally, (4) successfully prep and complete the colonoscopy. As such, the steps involved in this transfer from primary care to specialized medicine (e.g., GI) presents opportunities for patients to "fall through the cracks [9-11]." Furthermore, initial research conducted bivariate analyses to examine the factors (e.g., demographics) that were related to the likelihood to complete a referral to a specialist. The bivariate results found that some of the most vulnerable populations (e.g., patients in Medicaid plans, racial/ethnic minorities) may be the least likely to follow through with a primary care referral to a specialist [9].

It is critical to understand whether racial/ethnic minorities who are deemed ineligible for DAC are, in fact, complying with the PCPs recommendations and referrals. As such, the primary aim of the present study was to identify the proportion of racial/ethnic minority patients recommended to undergo screening colonoscopy but was ineligible for DAC, who, in fact, completed their screening colonoscopy via the GI referral. For the purpose of this study, we are predominantly focusing on patients who self-identify as African American or Latino.

The secondary aim was to examine predictors of nonadherence to screening colonoscopy recommendations in this population (i.e., racial/ethnic minorities deemed ineligible or DAC). Previous research suggests that demographic factors (e.g., age, sex, family history of CRC) [12-14] and psychological factors (e.g., fear of colonoscopy [15], fatalism [16], decisional balance of having CRC screening [17], CRC knowledge [18], and self-efficacy [19]) have been associated with non-adherence to CRC screening guidelines. Drawing from this research, it is hypothesized that these demographic and psychological factors will also be predictive of screening colonoscopy completion in racial/ethnic minorities who are ineligible for DAC.

\section{Methods}

Participants

All participants were enrolled in a larger study which examined the efficacy of a patient navigation intervention on adherence to physician-recommended screening colonoscopy $[20,21]$. The parent study had institutional review board approval. Eligibility criteria included (1) age 50 or older, (2) average risk for CRC, (3) attended a primary care non-acute medical visit, (4) received a PCP recommendation for a screening colonoscopy, and (5) had not had a screening colonoscopy within the last 5 years. Of the 1,303 patients recruited to the parent study, 1,183 (91\%) consented to participate. Of the enrolled participants, $144(12 \%)$ were ineligible for DAC and thus were referred for a pre-colonoscopy consultation with GI. Per medical chart review, the most frequently cited reason for DAC ineligibility was "multiple medical issues." The present study conducted secondary analyses on the subset of participants deemed ineligible for DAC and referred to the GI clinic $(N=144)$.

All data were collected with interviewer-administered questionnaires; all demographic information was self-report. The participants were predominantly female $(64 \%)$ and ranged in age from 49 to $81(M=61.6, \mathrm{SD}=7.7)$. At our institution, when patients are nearly 50 years old, PCPs may refer them for a screening colonoscopy and thus our sample included one participant who was 49 years old. Table 1 depicts additional demographic and medical information.

\section{Data Collection}

Eligible patients were recruited to the parent study immediately following their primary care appointment in which they received physician recommendation for a screening colonoscopy. Consented participants met with a research assistant in the private room in the primary care clinic to complete a 30 min verbally administered questionnaire that assessed the following: demographics (e.g., age, race), medical information (e.g., family history of CRC), and psychological variables (e.g., self-efficacy). Assessment interviews were conducted in either English or Spanish according to the participants' preference. Each participant was compensated $\$ 20.00$ for participation.

Within 1 week of the screening colonoscopy referral, a nurse in the Division of GI reviewed the enrolled participants' electronic medical charts to determine eligibility for DAC. If a participant was deemed ineligible for DAC, a note was made in the medical chart for the PCP to then refer the patient for a pre-colonoscopy GI consultation. As part of standard clinical procedures at our GI clinic, CRC screening information (e.g., colonoscopy preparation instructions) was provided in either English or Spanish by bilingual GI nursing staff. Furthermore, 
Table 1 Participant demographics

\begin{tabular}{ll}
\hline Demographic variable & $N(\%)$ \\
\hline Race/ethnicity & \\
African American & $58(40 \%)$ \\
Hispanic & $71(49 \%)$ \\
Other & $13(9 \%)$ \\
Unknown & $2(1 \%)$ \\
Insurance type & \\
Medicare & $53(37 \%)$ \\
Medicaid & $79(55 \%)$ \\
Private & $11(8 \%)$ \\
Unknown & $1(<1 \%)$ \\
Income & \\
$\leq \$ 10,000$ & $75(52 \%)$ \\
\$10,000-\$19,999 & $36(25 \%)$ \\
\$20,000-\$29,999 & $7(5 \%)$ \\
$\geq \$ 30,000$ & $14(10 \%)$ \\
Unknown & $12(8 \%)$ \\
Education & \\
$\leq 8$ th grade & \\
9th grade-12th grade & \\
12th grade-2 years college & \\
2 years college-4 years college & $33(23 \%)$ \\
Post college & $86(60 \%)$ \\
Fnknown & $19(13 \%)$ \\
Nes & $4(3 \%)$ \\
Unknown & $1(<1 \%)$ \\
\hline & $2(1 \%)$ \\
\hline
\end{tabular}

the CRC screening information was provided in a simple language so that individuals with varying reading levels can understand the materials. After completing the preconsultation visit with GI, participants were able to schedule and complete the recommended screening colonoscopy.

The participants deemed ineligible for DAC also became ineligible for participation in the parent study and thus did not receive any form of patient navigation. Electronic medical charts were reviewed 1 year following the primary care appointment to determine whether the participants attended GI and subsequently completed the recommended screening colonoscopy.

\section{Measures}

Demographics/Medical Information A sociodemographics/ medical questionnaire assessed self-reported demographic and medical information including age, race/ethnicity, employment status, income, education, and family history of cancer and insurance type.
Fear of Colonoscopy Manne's Fear of Colonoscopy Scale [22] was used to assess the fear of colonoscopy (personal communication). Participants were asked to rate their level of fear associated with certain components of the colonoscopy procedure (e.g., possible pain during the procedure). The six items were rated on a five-point Likert scale ranging from 1 (not at all fearful) to 5 (extremely fearful). A mean score was calculated, where higher scores represent higher levels of fear. In the current subsample, the scale's internal consistency was acceptable (Cronbach's alpha=0.83).

Fatalism A five-item measure, adapted from the Powe Fatalism Inventory [16], was used to assess fatalistic thoughts (e.g., "I believe if someone gets cancer, their time to die is near"). Participants indicated their agreement with each item (yes/no). A mean score was calculated, where higher scores represent higher levels of fatalism. In the current subsample, the scale's internal consistency was acceptable (Cronbach's alpha=0.84).

Decisional Balance A seven-item measure, adapted from Manne's pros/cons of screening scale [22], was used to assess perceived benefits and barriers of having a colonoscopy. Three items described perceived benefits of having a colonoscopy (e.g., Having a regular colonoscopy would give me peace of mind about my health), and four items described perceived barriers of having a colonoscopy (e.g., Having a colonoscopy is very embarrassing). Participants indicated their agreement with each item on a five-point Likert scale ranging from 1 (strongly disagree) to 5 (strongly agree). The mean benefit score was subtracted from the mean barrier score to create a total decisional balance score. Higher scores represent higher perceived barriers, relative to perceived benefits. In the current subsample, the mean barrier subscale and the mean benefit subscales had suboptimal internal consistencies (Cronbach's alphas $=0.59$ and 0.42 , respectively).

CRC Knowledge A four-item measure, drawn from prior literature [22], used true-false statements to assess participants' level of knowledge about CRC risk (e.g., "A person could have colorectal cancer without having any symptoms"). The percentage of total correct responses was calculated to generate an overall CRC knowledge score where higher scores represent greater knowledge. In the current subsample, the scale had suboptimal internal consistency (Cronbach's alpha=0.43).

Self-efficacy A ten-item measure, adapted from previous literature on mammography [23], was used to assess participants' belief in their ability to complete a screening colonoscopy. Participants were asked to report how confident they felt about performing certain tasks (e.g., "I can get a colonoscopy even if I don't know what to expect"). Items were ranked on a five-point Likert scale ranging from 1 (strongly disagree) to 5 
(strongly agree). A mean self-efficacy score was calculated where higher scores represent greater levels of self-efficacy. In the current subsample, the scale's internal consistency was acceptable (Cronbach's alpha=0.87).

\section{Results}

The primary aim of the study was to determine the proportion of participants ineligible for DAC that adhered to a PCP's recommendation for a GI consult and screening colonoscopy. Descriptive statistics revealed that of the 144 participants referred to GI, only $32(22 \%)$ attended the appointment with GI and 19 (13\% of the total) completed their screening colonoscopy. Of note, within 1 year of the original screening colonoscopy referral, six participants returned to their PCP for a subsequent visit and eventually completed the screening colonoscopy via direct access. Thus, as shown in Fig. 1, only $25(17 \%)$ of the participants completed the recommended screening colonoscopy procedure within 1 year of the initial referral (including the six participants who completed the screening colonoscopy via direct access).
Predictors of Colonoscopy Adherence

The secondary aim of the study was to determine baseline predictors of screening colonoscopy adherence, through the GI referral process. As such, the six participants who eventually completed the screening colonoscopy via direct access were coded as "non-adherent" because they failed to complete the colonoscopy via the GI referral process.

First, bivariate analyses were run to determine the variables that were significantly related to screening colonoscopy adherence. Then, the significant correlates were entered into a final regression model predicting screening colonoscopy adherence. Bivariate analyses revealed that demographic variables (i.e., age, education, income) were not significantly related to screening colonoscopy adherence. For the question regarding income, 12 participants refused to answer. For the bivariate correlation between income and colonoscopy completion, those 12 participants were excluded from the analysis. In addition, chi-square analyses revealed that family history of cancer, insurance type, nor gender were related to screening colonoscopy adherence ( $p$ values $>.1$ ). Of the measured psychological predictors, decisional balance $(p<.001)$ and selfefficacy $(p<.01)$ were the only significant correlates of colonoscopy adherence (see Table 2 ).
Fig. 1 Participants' adherence to GI and colonoscopy referrals

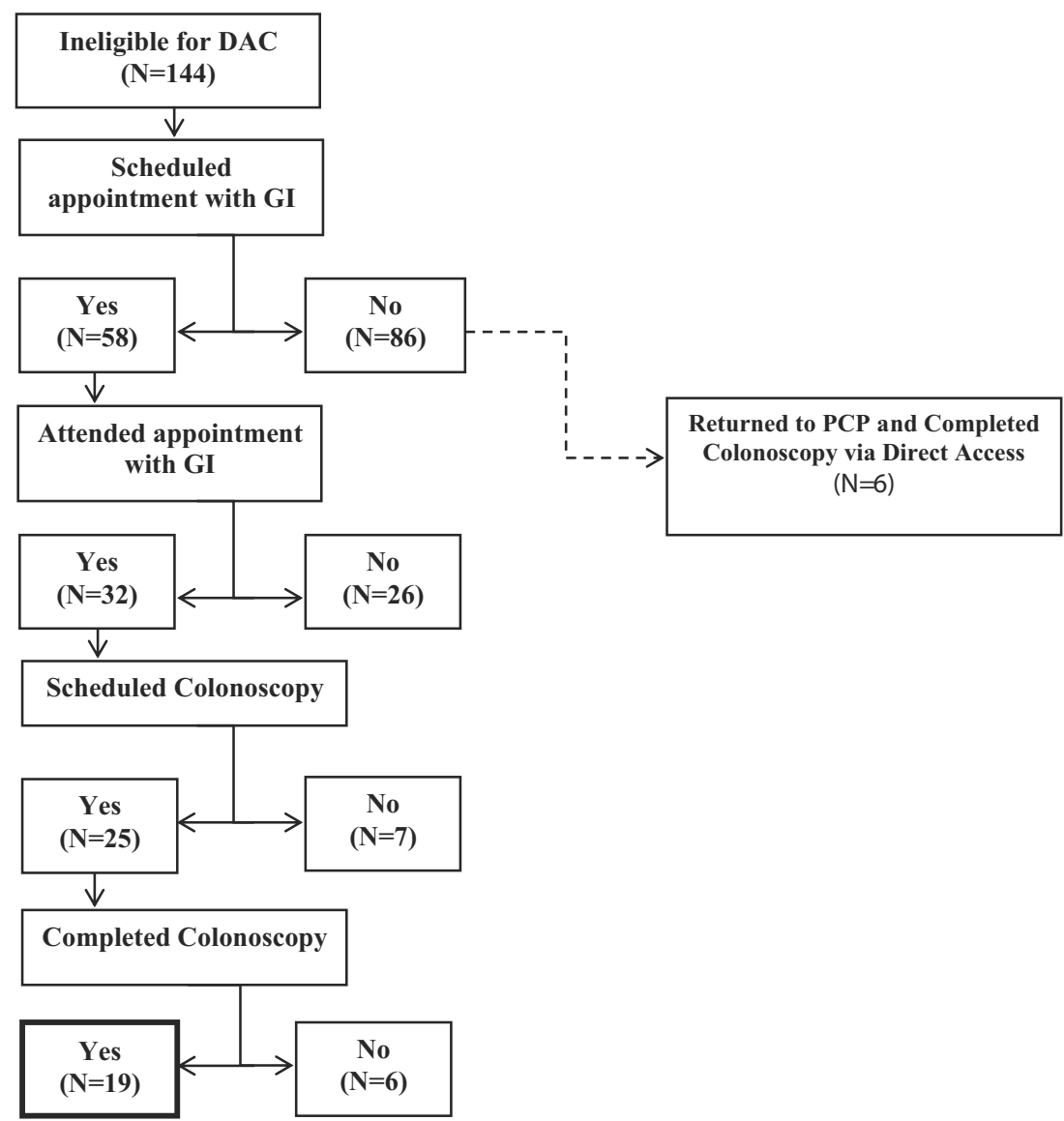


Table 2 Bivariate correlation table

\begin{tabular}{lll}
\hline & Complete screening colonoscopy $^{\mathrm{a}}$ & $p$ value \\
\hline Fear of colonoscopy & -0.09 & 0.26 \\
Fatalism & -0.07 & 0.43 \\
Decisional balance & -0.31 & $<0.00$ \\
Knowledge of CRC & 0.03 & 0.72 \\
Self-efficacy & 0.22 & 0.01 \\
\hline
\end{tabular}

${ }^{a}$ Note: The colonoscopy completion outcome solely included participants who completed the screening via the GI referral

Next, the significant correlates were entered into a multivariate regression model. In particular, self-efficacy and decisional balance were entered into a logistic regression in order to identify unique predictors of screening colonoscopy adherence. The overall model was significant $\chi^{2}(2, N=143)=16.79$ and explained between $11 \%$ (Cox and Snell R Square) and $20 \%$ (Nagelkerke R Square) of the variance. The results revealed that decisional balance was the only variable to uniquely predict screening colonoscopy adherence $(\mathrm{OR}=0.34 ; 95 \%$ $\mathrm{CI}=0.16,0.73)(p<.01)$.

\section{Discussion}

The current study suggests that approximately one in every nine average-risk racial/ethnic minority patients referred for a screening colonoscopy is deemed ineligible for DAC. As part of the standard clinical practice, these patients are recommended to attend a pre-colonoscopy consultation with GI. The present results revealed that this transfer from primary care to GI creates a window for patients to drop-out of treatment. In the current sample, only $22 \%$ of the participants attended the recommended pre-colonoscopy clinic visit with GI. Of additional concern, among the participants who attended a GI visit, less than 2/ 3 subsequently completed the recommended screening colonoscopy. Thus, overall, only $13 \%$ of the participants had a screening colonoscopy via the GI referral process.

Our screening colonoscopy completion rates $(13 \%)$ are considerably lower than the screening colonoscopy completion rates reported in the literature. For example, the CDC reports that $60.2 \%$ of the US population has received a screening colonoscopy within the recommended 10-year time frame [24]. These contrasting results highlight that racial/ethnic minorities ineligible for DAC are a particularly high-risk population. It is possible that these patients face unique barriers to completing a screening colonoscopy (e.g., difficulty managing multiple medical conditions). Future comparative studies should examine the differences between this population and the general population.

In addition, the contrast between the screening colonoscopy completion rates reported in our study and the rates reported in the literature suggest that a proportion of the participants in our sample (i.e., racial/ethnic minorities ineligible for DAC) may eventually receive a screening colonoscopy within a 10 -year time frame. In fact, within the 1-year timeframe of our study, six participants returned to their PCP were then deemed eligible for DAC and eventually completed the colonoscopy. Drawing from these results, it is likely that at least some patients ineligible for DAC will be re-referred for a screening colonoscopy and eventually complete the procedure.

The low screening rates $(13 \%)$ are particularly disheartening when compared to the screening colonoscopy adherence rates among racial/ethnic minorities in our parent study who were eligible for DAC and received patient navigation. In particular, in the parent study, participants who received patient navigation had screening colonoscopy completion rates ranging between 74 and $81 \%[20,21]$. There were no statistical differences in completion rates based on the type of navigation (e.g., peer navigation, standard navigation) the participants received. The results of the parent study highlight the capability of patient navigation to improve racial/ ethnic minorities' screening colonoscopy completion rates. There is a great need to apply these types of navigation interventions $[21,25]$ to help racial/ethnic minorities ineligible for DAC better navigate the referral from primary care to GI and subsequently complete the screening colonoscopy.

The current study also examined the intrapersonal variables that are predictive of screening colonoscopy adherence in racial/ethnic minorities ineligible for DAC. Consistent with previous research [17], the current study revealed that participants with higher scores on a decisional balance scale were at greatest risk for colonoscopy non-adherence. That is, participants who reported higher perceived barriers to having a colonoscopy, relative to perceived benefits were less likely to complete the colonoscopy. Interventions designed to improve decisional balance, such as motivational interviewing [26-28], can be integrated into patient navigation programs to directly address this barrier.

In addition to intrapersonal barriers (i.e., decisional balance), it is possible that institutional and system barriers are playing a role in this population's low screening colonoscopy adherence rates $(13 \%)$. For example, when patients are deemed ineligible for DAC, it is the PCP's responsibility to notify the patients about the required pre-colonoscopy GI consultation. Many factors (e.g., patients' ability to be reached, patient-physician communication) may impact the 
likelihood that patients receive the referral. Then, after the patients receive a referral, they are required to schedule an appointment with GI and a subsequent appointment for screening colonoscopy. The clinic's availability and wait times, for example, may further impact the likelihood that patients will eventually be screened. Because there are multiple barriers to screening colonoscopy uptake, we recommend that patients ineligible for DAC receive patient navigation in order to help them overcome intrapersonal (e.g., poor decisional balance), physician (e.g., physician-patient communication), and system (e.g., long wait times to schedule appointments) barriers to receiving a screening colonoscopy.

There are limitations to the current study that should be taken into account when interpreting and applying the findings. First, the regression model only accounted for $11-20 \%$ of the variance in screening colonoscopy adherence rates. Future research should examine additional barriers and facilitators to screening colonoscopy adherence in racial/ethnic minorities deemed ineligible for DAC. For example, research should examine the role of health literacy in predicting screening uptake in this at-risk population as previous studies have found that poor health literacy is a barrier to CRC screening uptake [29-31]. Second, the sample size was small and it is possible that larger sampled studies would be able to better detect associations between the predictors and outcomes. Third, the reliability coefficients of the measures of decisional balance and CRC knowledge were less than desirable. It is likely that the low Cronbach's alphas are partially an artifact of the small number of items in the measures [32]. In designing the study, some standard measures were shortened in order to minimize participant burden. Future research should replicate this study with longer, more comprehensive measures of CRC knowledge and decisional balance in order to confirm the study results.

\section{Conclusions}

Although screening colonoscopies can aid in the early detection and prevention of CRC, a sizable proportion of patients remain unscreened [1, 24]. In recent years, the New York C5 Coalition and the NYC DOHMH have made great strides toward improving racial/ethnic minorities' participation in screening colonoscopies by implementing Direct Referral Initiative for screening colonoscopies. Unfortunately, the DAC referral system is not available or appropriate for all patients. As demonstrated by the current study, patients deemed ineligible for DAC seem to be falling through the system's cracks and, as a result, are at considerable risk for non-adherence to screening colonoscopy recommendations. Interventions are needed to help this vulnerable population navigate the referral from primary care to GI with the ultimate goal of detecting and preventing CRC.

Acknowledgments Preparation of this manuscript was supported by the National Cancer Institute (R01CA120658; R25CA081137) and the American Cancer Society (122931-PF-12-117-01-CPPB). The content is solely the responsibility of the authors and does not necessarily represent the official views of the National Cancer Institute or the National Institutes of Health.

Conflict of Interest Sarah Miller, Jamilia Sly, Steven Itzkowitz, and Lina Jandorf declare that they have no conflict of interest.

Informed Consent All procedures followed were in accordance with the ethical standards of the responsible committee on human experimentation (institutional and national) and with the Helsinki Declaration of 1975, as revised in 2000 (5). Informed consent was obtained from all patients for being included in the study.

Animal Studies No animal or human studies were carried out by the authors for this article.

\section{References}

1. American Cancer Society. Colorectal cancer facts \& figures 20112013. 2011:1-27.

2. Preventive Services US. Task Force. Screening for colorectal cancer: U.S. Preventive Services Task Force recommendation statement. Ann Intern Med. 2008;149(9):627-37.

3. Levin B, Lieberman DA, McFarland B, Andrews KS, Brooks D, Bond J, et al. Screening and surveillance for the early detection of colorectal cancer and adenomatous polyps, 2008: a joint guideline from the American Cancer Society, the US Multi-Society Task Force on Colorectal Cancer, and the American College of Radiology. Gastroenterology. 2008;134(5):1570-95. doi:10.1053/j.gastro.2008. 02.002 .

4. Rex DK. Colonoscopy: the dominant and preferred colorectal cancer screening strategy in the United States. Mayo Clin Proc. 2007;82(6): 662-4. doi: $10.4065 / 82.6 .662$.

5. Sifri R, Wender R, Lieberman D, Potter M, Peterson K, Weber TK, et al. Developing a quality screening colonoscopy referral system in primary care practice: a report from the national colorectal cancer roundtable. CA Cancer J Clin. 2010;60(1):40-9. doi:10.3322/caac. 20048.

6. Mahajan RJ, Marshall JB. Prevalence of open-access gastrointestinal endoscopy in the United States. Gastrointest Endosc. 1997;46(1):21-6.

7. New York City Department of Health and Mental Hygiene. Colonoscopy Direct Referral Initiative. 11/27/12; Available at: http://www.nyc.gov/html/doh/html/living/cancer-colon-providerreferral.shtml. Accessed 27 May 2014.

8. Chen LA, Santos S, Jandorf L, Christie J, Castillo A, Winkel G, et al. A program to enhance completion of screening colonoscopy among urban minorities. Clin Gastroenterol Hepatol. 2008;6(4):443-50. doi: 10.1016/j.cgh.2007.12.009.

9. Forrest CB, Shadmi E, Nutting PA, Starfield B. Specialty referral completion among primary care patients: results from the ASPN Referral Study. Ann Fam Med. 2007;5(4):361-7.

10. Byrd JC, Moskowitz MA. Outpatient consultation: interaction between the general internist and the specialist. J Gen Intern Med. 1987;2(2):93-8. 
11. Bourguet C, Gilchrist V, McCord G. The consultation and referral process. A report from NEON. Northeastern Ohio Network Research Group. J Fam Pract. 1998;46(1):47-53.

12. Seeff LC, Nadel MR, Klabunde CN, Thompson T, Shapiro JA, Vernon SW, et al. Patterns and predictors of colorectal cancer test use in the adult U.S. population. Cancer. 2004;100(10):2093-103.

13. Denberg TD, Melhado TV, Coombes JM, Beaty BL, Berman K, Byers TE, et al. Predictors of nonadherence to screening colonoscopy. J Gen Intern Med. 2005;20(11):989-95. doi:10.1111/j.15251497.2005.00164.x.

14. Subramanian S, Klosterman M, Amonkar MM, Hunt TL. Adherence with colorectal cancer screening guidelines: a review. Prev Med. 2004;38(5):536-50. doi:10.1016/j.ypmed.2003.12.011.

15. Jandorf L, Ellison J, Villagra C, Winkel G, Varela A, Quintero-Canetti $\mathrm{Z}$, et al. Understanding the barriers and facilitators of colorectal cancer screening among low income immigrant Hispanics. J Immigr Minor Health. 2010;12(4):462-9. doi:10.1007/s10903-009-9274-3.

16. Powe BD. Fatalism among elderly African Americans. Effects on colorectal cancer screening. Cancer Nurs. 1995;18(5):385-92.

17. Lawsin C, DuHamel K, Weiss A, Rakowski W, Jandorf L. Colorectal cancer screening among low-income African Americans in East Harlem: a theoretical approach to understanding barriers and promoters to screening. J Urban Health. 2007;84(1):32-44. doi:10.1007/ s11524-006-9126-6.

18. Harewood GC, Wiersema MJ, Melton 3rd LJ. A prospective, controlled assessment of factors influencing acceptance of screening colonoscopy. Am J Gastroenterol. 2002;97(12):3186-94. doi:10. 1111/j.1572-0241.2002.07129.x.

19. McQueen A, Vernon SW, Myers RE, Watts BG, Lee ES, Tilley BC. Correlates and predictors of colorectal cancer screening among male automotive workers. Cancer Epidemiol Biomarkers Prev. 2007;16(3):500-9. doi:10.1158/1055-9965.EPI-06-0757.

20. Jandorf L, Braschi C, Ernstoff E, Wong CR, Thelemaque LD, Winkel $\mathrm{G}$, et al. Culturally targeted patient navigation for increasing African American's adherence to screening colonoscopy. Cancer Epidemiol Biomarkers Prev. 2013;22(9):1577-87. doi:10.1158/1055-9965.EPI12-1275.

21. Braschi CD, Sly JR, Singh S, Villagra C, Jandorf L. Increasing colonoscopy screening for Latino Americans through a patient navigation model: a randomized clinical trial. J Immigr Minor Health. 2013. doi:10.1007/s10903-013-9848-y.

22. Manne S, Markowitz A, Winawer S, Meropol NJ, Haller D, Rakowski W, et al. Correlates of colorectal cancer screening compliance and stage of adoption among siblings of individuals with early onset colorectal cancer. Health Psychol. 2002;21(1):3-15.

23. Champion V, Skinner CS, Menon U. Development of a self-efficacy scale for mammography. Res Nurs Health. 2005;28(4):329-36. doi: 10.1002/nur.20088.

24. Steele CB, Rim SH, Joseph DA, King JB, Seeff LC. National Center for Chronic Disease Prevention and Health Promotion, CDC. Colorectal cancer incidence and screening-United States, 2008 and 2010. MMWR Surveill Summ. 2013;62 Suppl 3:53-60.

25. Nash D, Azeez S, Vlahov D, Schori M. Evaluation of an intervention to increase screening colonoscopy in an urban public hospital setting. J Urban Health. 2006;83(2):231-43. doi:10.1007/s11524-006-90296.

26. Apodaca TR, Longabaugh R. Mechanisms of change in motivational interviewing: a review and preliminary evaluation of the evidence. Addiction. 2009;104(5):705-15. doi:10.1111/j.1360-0443.2009. 02527.x.

27. Martins RK, McNeil DW. Review of motivational interviewing in promoting health behaviors. Clin Psychol Rev. 2009;29(4):283-93.

28. Emmons KM, Rollnick S. Motivational interviewing in health care settings. Opportunities and limitations. Am J Prev Med. 2001;20(1): 68-74.

29. Kobayashi LC, Wardle J, von Wagner C. Limited health literacy is a barrier to colorectal cancer screening in England: evidence from the English Longitudinal Study of Ageing. Prev Med. 2014;61:100-5. doi:10.1016/j.ypmed.2013.11.012.

30. Sentell T, Braun KL, Davis J, Davis T. Colorectal cancer screening: low health literacy and limited English proficiency among Asians and Whites in California. J Health Commun. 2013;18 Suppl 1:242-55. doi:10.1080/10810730.2013.825669.

31. Wang J, Moehring J, Stuhr S, Krug M. Barriers to colorectal cancer screening in Hispanics in the United States: an integrative review. Appl Nurs Res. 2013;26(4):218-24. doi:10.1016/j.apnr.2013.08.005.

32. Tavakol M, Dennick R. Making sense of Cronbach's alpha. Int J Med Educ. 2011;2:53-5. doi:10.5116/ijme.4dfb.8dfd. 